\title{
Identification of Evolutionary Hotspots in the Rodent Genomes
}

\author{
Von Bing Yap ${ }^{1}$ and Lior Pachter \\ Department of Mathematics, University of California, Berkeley, California 94720-3840, USA
}

\begin{abstract}
We describe a whole-genome comparative analysis of the human, mouse, and rat genomes to describe the average substitution patterns of four genomic regions: ancient repeats, rodent-specific DNA, exons, and conserved (coding and noncoding) regions, and to identify rodent evolutionary hotspots. In all types of regions, except the rodent-specific DNA, the rat branch is slightly longer than the mouse branch. Moreover, the mouse-rat distance is longer in the rodent-specific DNA than in the ancient repeats. Analysis of individual conserved regions with different substitution models yielded the conclusion that the Jukes-Cantor model is inadequate, and the Hasegawa-Kishino-Yano model is almost as good as the REV model. Using human as an outgroup, we identified 5055 evolutionary hotspots, which are highly conserved subalignment blocks (each consisting of at least 100 aligned sites and a small fraction of gaps) with a large and statistically significant difference in the branch lengths of the rodent species. The cutoffs used to identify the hotspots are partially based on estimates of the average rates of substitution. The fractions of hotspots overlapping with the rodent RefSeq genes, RefSeq exons, and ESTs are all higher than expected. Still, more than half of the hotspots lie in noncoding regions of the mouse genome. We believe that the hotspots represent biologically interesting regions in the rodent genomes.
\end{abstract}

The sequencing of the rat genome makes possible, for the first time, a whole-genome comparative analysis of three large mammalian genomes. Despite the exciting prospects of such an analysis, existing methods on a whole (mammalian) genome scale are scarce. Some examples include methods based on gene order comparison rather than sequence comparison (Moret et al. 2001). One of the difficulties in whole-genome comparison is that it is necessary to begin with a reliable multiple alignment of the genomes. Even pairwise whole-genome comparison is difficult (Wiehe et al. 2000; Miller 2001), and the addition of genomes significantly complicates the alignment problem with resulting consequences for the inferences that are to be made.

Three species comparison of genomic regions was first undertaken by Lee et al. (1998). Subsequent analysis of three species data (Dubchak et al. 2000) for the identification of actively conserved non-coding regions resulted in suggested percent identity cutoffs for extracting functional regions from human, mouse, and dog alignments. Recent targeted sequencing projects have yielded larger data sets for analysis (Boffelli et al. 2003; Cooper et al. 2003; Thomas et al. 2003), revealing the power of comparative analysis both for understanding sequence evolution and for identifying functional elements.

We performed a comparative analysis on a whole-genome multiple alignment of human, mouse, and rat DNA sequences, to describe the average substitution patterns of four types of DNA: ancient repeats, rodent-specific DNA, exons, and conserved regions (coding and noncoding), and to identify rodent evolutionary hotspots, which are well-conserved regions where the rodent branch lengths are very different. The data were obtained by extracting appropriate gapped subalignments, called blocks, from the whole-genome alignment. The blocks were aggregated to estimate the average substitution rates and branch lengths of the unrooted tree relating the three species (Fig. 1) for each type of DNA, by maximum likelihood on the REV substitution model

\section{'Corresponding author.}

E-MAIL vonbing@math.berkeley.edu; FAX (510) 642-8204.

Article and publication are at http://www.genome.org/cgi/doi/10.1101/ gr.1967904.
(Tavaré 1986; Yang 1994). Then we investigated the effect of substitution model on branch length estimation applied to individual conserved regions. Finally, rodent evolutionary hotspots were selected from the conserved regions based on criteria that are partially motivated by the average substitution patterns.

The ancient repeats (Waterston et al. 2002; Hardison et al. 2003) and rodent-specific DNA, which do not align to any human DNA (Cooper et al. 2003a), are believed to be enriched in nonfunctional sequences, hence likely to be undergoing neutral evolution. A human repetitive element, which was neither simple nor low complexity, was selected if at least $80 \%$ of its bases were aligned to both mouse and rat. Such an alignment is likely to be a good alignment between repetitive elements that descended from the same ancestor before speciation. Rodentspecific DNA were collected by selecting blocks with at least $a=50$ aligned sites, flanked by gaps of length greater than $g=5$, and containing gaps of length at most $g=5$, with total length at most $10 \%$ of the number of aligned sites from genomic regions where rodent DNAs align to gaps in the human sequence. The conservative criteria ensured that the selected blocks had a small number of short gaps, so that we were confident that the alignments were solid. For exons, we selected human RefSeq exons with at least $80 \%$ of their bases aligned to both mouse and rat. The rationale is similar to that for ancient repeats. Finally, the criteria for conserved regions are very similar to those for rodentspecific DNA, with $a=100$ and $g=10$. We required at least 100 aligned sites so that the branch lengths can be estimated reliably from individual blocks. In addition, we only selected blocks where all three pairwise similarities exceeded $60 \%$, to save computation time and to sift out spurious alignments. As will be seen, this extra requirement has little effect on the results. Although the filters are largely heuristic and arbitrary, they are conservative in the sense that most of the selected blocks are believed to represent real alignments of conserved sequences from all three species.

An evolutionary hotspot is a conserved region with the property that the human branch is shorter than 0.25 substitutions per site, the absolute difference between the rodent branch lengths is more than twice the asymptotic standard error, and the 


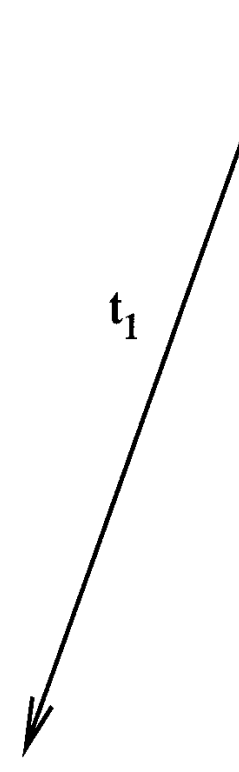

h

Figure 1 The evolutionary tree relating $\mathbf{h}, \mathbf{m}, \mathbf{r}$, the most recent rodent ancestor $\mathbf{y}$, and the most recent common ancestor $\mathbf{z}$. By pooling ancient repeat sites, the branch lengths are $t_{h}=t_{1}+t_{2}=0.36, t_{m}=t_{3}=0.070$ and $t_{r}=t_{4}=0.073$ substitutions per site.

ratio of the mouse branch to the rat branch is at least 10 or at most 0.1 . These are well-conserved regions, likely to contain functional elements, where the rodents have evolved at very different rates.

\section{RESULTS}

\section{Average Substitution Patterns}

Table 1 displays some statistics and the branch lengths for four types of regions: ancient repeats, rodent-specific DNA, exons, and conserved regions; the substitution patterns were aggregated in these analyses. Because the rodent-specific blocks are really pairwise alignments, the distance between the rodents, but not their respective distances to the rodent ancestor, may be estimated via the REV model. For all other regions, the mouse branch is slightly shorter than the rat branch, the ratios ranging from 0.91 to 0.95 , indicating that mouse generally evolved slightly more slowly, relative to rat. The fractions of blocks with the rat branch longer than the mouse branch were $54 \%$ for long ancient repeats ( $>100$ aligned sites), $52 \%$ for long exons ( $>100$ aligned sites), and $55 \%$ for conserved regions. The ratio of the human branch to the mouse branch is $\sim 5$. These are consistent with observations by others (Cooper et al. 2004; Yang et al. 2004). Ranking by any branch length gives the expected ordering

$$
\text { Exon }<\text { Conserved }<\text { AR, }
$$

because, in general, exons evolved under a lot of selective pressure, whereas the ancient repeats are likely to be enriched in nonfunctional DNA, and conserved regions are intermediate. Ordering by the mouse-rat distance yields

$$
\mathrm{AR}<\text { Rodent. }
$$

Because the amount of data is so large, the discrepancy cannot be explained by statistical fluctuations but, rather, indicates a real difference. The orderings are quite robust to mild perturbations in the selection criteria. For example, the mouse-rat distances are 0.13 (at 90\% threshold) and $0.15(70 \%)$ for AR; $0.17(a=100$, $g=10)$ and $0.18(a=20, g=2)$ for rodent-specific DNA; 0.06 (90\%) and 0.08 (70\%) for exons; and 0.09 (70\%), 0.10 (50\%), and $0.12(0 \%)$ for conserved regions. Both the parameter values $a=20, g=2$, and the mouse-rat distance 0.18 are very similar to those by Cooper et al. (2004). Perturbing other parameters for conserved regions still gave estimates within the range.

The estimated REV rate matrices are shown in Table 2 . All rate matrices are not of the HKY type, $R(C, A)$ being typically larger than $R(T, A)$; the exon rate matrix is the closest to HKY. However, they are very close to being strand-symmetric, that is, the rates are invariant under complementation. For example, $Q(A, C) \sim Q(T, G)$. The AR and Rodent rates are remarkably similar, and they are both similar to the rates for conserved regions. Finally, all the rate matrices are more similar to each other than to those corresponding to the $4 \mathrm{D}$ sites and ancient repeats in Hardison et al. (2003). This could be caused by differences in data, alignment, methodology, or other factors.

\section{Sensitivity of Branch Length Estimates to Substitution Model}

The average substitution patterns were studied using aggregated blocks, in which blocks of any size were effectively glued together to form a large block. On the other hand, to identify interesting regions for further study, it is necessary to apply the estimation procedure to individual blocks, which were required to have at least 100 aligned sites so that the estimates were not too variable. We compare the branch lengths of 646,741 conserved regions estimated via the Jukes-Cantor (JC), HasegawaKishino-Yano (HKY), and general reversible (REV) models by maximum likelihood. Between JC and REV, the fractions of blocks for which the difference is $<0.01$ is $85 \%$ (rodents) and $27 \%$ (human). The corresponding fractions for comparing HKY and REV are 94\% (rodents) and 79\% (human). Thus, HKY is significantly more accurate than JC. Generally, the REV estimates are larger than the HKY estimates, which are, in turn, larger than the JC estimates. Also, the difference between REV and HKY (also REV and JC) tends to decrease modestly as the branch length (with the REV estimate as a proxy) decreases (see Fig. 2), which is consistent with 
Table 2. Estimated REV Rate Matrix, $Q$, Its Symmetric Part, $R$, and Its Equilibrium Distribution $\pi$, for Four Types of Regions

\begin{tabular}{|c|c|c|c|c|c|c|c|c|c|}
\hline \multirow{2}{*}{$\begin{array}{l}\text { Region } \\
A R\end{array}$} & \multicolumn{4}{|c|}{$Q$} & \multicolumn{4}{|c|}{$R$} & \multirow{2}{*}{$\frac{\pi}{0.29}$} \\
\hline & -0.87 & 0.18 & 0.52 & 0.18 & -2.97 & 0.84 & 2.52 & 0.60 & \\
\hline & 0.25 & -1.17 & 0.19 & 0.74 & 0.84 & -5.64 & 0.90 & 2.52 & 0.21 \\
\hline & 0.74 & 0.19 & -1.18 & 0.26 & 2.52 & 0.90 & -5.74 & 0.87 & 0.21 \\
\hline & 0.18 & 0.52 & 0.18 & -0.88 & 0.60 & 2.52 & 0.87 & -3.00 & 0.29 \\
\hline \multirow[t]{4}{*}{ Rodent } & -0.88 & 0.17 & 0.53 & 0.19 & -3.03 & 0.83 & 2.53 & 0.63 & 0.29 \\
\hline & 0.24 & -1.16 & 0.18 & 0.74 & 0.83 & -5.55 & 0.84 & 2.54 & 0.21 \\
\hline & 0.74 & 0.18 & -1.16 & 0.25 & 2.53 & 0.84 & -5.56 & 0.84 & 0.21 \\
\hline & 0.19 & 0.53 & 0.18 & -0.89 & 0.63 & 2.54 & 0.84 & -3.06 & 0.29 \\
\hline \multirow[t]{4}{*}{ Exon } & -1.05 & 0.19 & 0.71 & 0.15 & -4.53 & 0.72 & 2.60 & 0.64 & 0.23 \\
\hline & 0.17 & -0.96 & 0.18 & 0.61 & 0.72 & -3.55 & 0.64 & 2.68 & 0.27 \\
\hline & 0.60 & 0.17 & -0.95 & 0.17 & 2.60 & 0.64 & -3.48 & 0.75 & 0.27 \\
\hline & 0.15 & 0.72 & 0.20 & -1.07 & 0.64 & 2.68 & 0.75 & -4.69 & 0.23 \\
\hline \multirow[t]{4}{*}{ Conserved } & -0.89 & 0.18 & 0.54 & 0.18 & -3.09 & 0.83 & 2.51 & 0.61 & 0.29 \\
\hline & 0.24 & -1.14 & 0.19 & 0.72 & 0.83 & -5.35 & 0.88 & 2.51 & 0.21 \\
\hline & 0.72 & 0.19 & -1.15 & 0.24 & 2.51 & 0.88 & -5.40 & 0.85 & 0.21 \\
\hline & 0.18 & 0.54 & 0.18 & -0.89 & 0.61 & 2.51 & 0.85 & -3.12 & 0.29 \\
\hline
\end{tabular}

the fact that the variance is larger for a longer branch. Interestingly, for very short branches, the HKY and JC estimates tend to be larger than REV. This phenomenon may partially account for the fact that the number of hotspots increases as the substitution model becomes more accurate: 3672 (JC), 4522 (HKY), and 5055 (REV). We conclude that JC should be avoided, and although HKY may be adequate for branch length estimation, the REV model is better and thus we used the latter.

\section{Evolutionary Hotspots}

We found 5055 evolutionary hotspots among the conserved regions. The average and standard deviation (SD) of the number of aligned sites are 190 and 86, respectively (histogram in Fig. 3). If the conserved regions were not filtered by the requirement that the three pairwise similarities were at least $60 \%$, then the number of hotspots found was 5086. Thus, the effect of the filter is rather small for identification of hotspots.

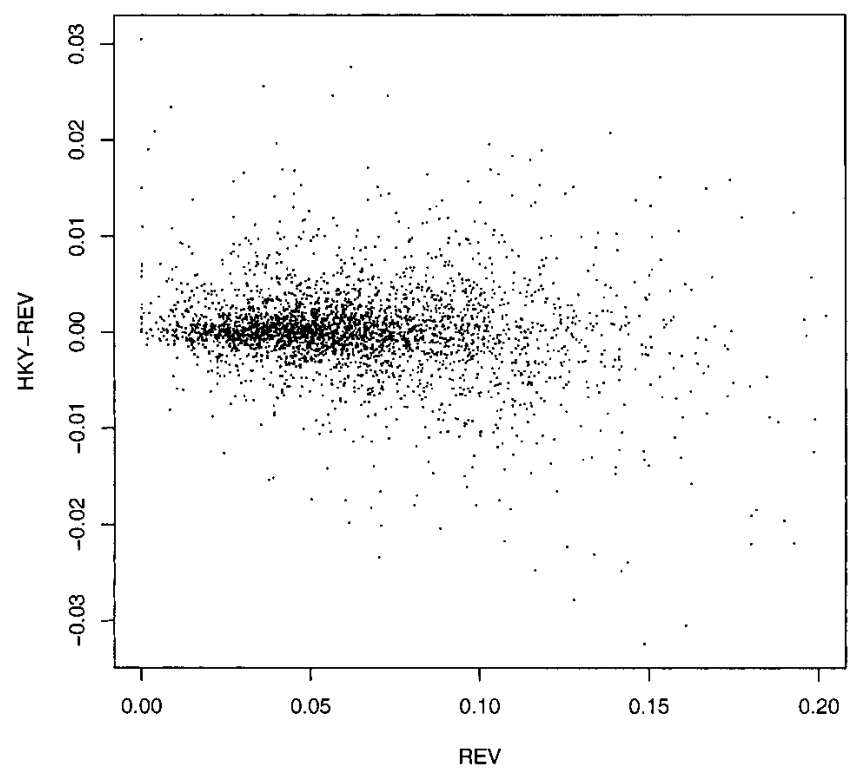

Figure 2 Plot of the difference of estimated rat branch length by the HKY and the REV against the REV branch length for the conserved regions. To present the data clearly, every 200 -th block was actually plotted, yielding 3234 points. The correlation coefficient for the plot is -0.17 , and for the 646,741 conserved regions is -0.18 . The corresponding plot for mouse is very similar.
Small fractions of the hotspots have some overlap with ancient repeats (mouse 6\%, rat 5\%) and simple and low-complexity repeats (mouse 9\%, rat $8 \%$ ). We also computed the fractions that have some overlap with RefSeq genes, RefSeq exons, and ESTs. Treating hotspots as points on the genome, if they were scattered randomly, then we would expect the fraction that landed on, say, the ESTs, to be roughly the fraction of ESTs in the genome. The observed and expected fractions are reported in Table 3 . The hotspots are overrepresented in the three regions by factors ranging from 2.0 (mouse ESTs) to 6.7 (rat exons). This observation still holds for each individual chromosome. Because the mouse RefSeq database is more complete, we infer that about $\sim 37 \%$ of the hotspots lie totally in the noncoding portion of mouse genes. Assuming that the mouse ESTs cover all RefSeq genes, $\sim 27 \%$ are intergenic in the mouse genome. Thus, $64 \%$, or more than half, of the hotspots are probably functional noncoding sequences in the mouse genome.

The evolutionary hotspots are available for downloading at http://baboon.math.berkeley.edu/hotrodent/. For long hotspots (>300 aligned sites, say), it is likely that the branch lengths vary considerably along the alignment. It is then desirable to perform the estimation on a sliding window for detailed study. Although it is difficult to characterize the hotspots in an automated fashion, examples we have analyzed are yielding seemingly interesting biological stories (although ones that may be difficult to verify by experiment). An example is a 505-base-long hotspot in the third intron of PEX14 (Rat Genome Sequencing Project Consortium 2004), described as peroxisomal biogenesis factor 14 in the RefSeq database. This nonrepetitive region shows remarkable heterogeneity in evolutionary rates; the 5 '-end has a very short mouse branch and a long rat branch, while near the 3 '-end, there is a stretch of 210 sites that are identical in all three species.

\section{DISCUSSION}

The sequencing of the rat genome has provided us, for the first time, the opportunity to compare and contrast closely related vertebrate genomes. We have specifically used the human genome as an outgroup to the rodent genomes to identify evolutionary hotspots; it is important to note that this analysis would not have been possible without the complete sequence for all three genomes. Although the phylogenetic tree for the human, mouse, and rat is rather simple, the estimation of the branch lengths is not, and, as we have shown, several interesting results emerge from a detailed analysis of the branch length estimates and their sensitivity to parameters. 


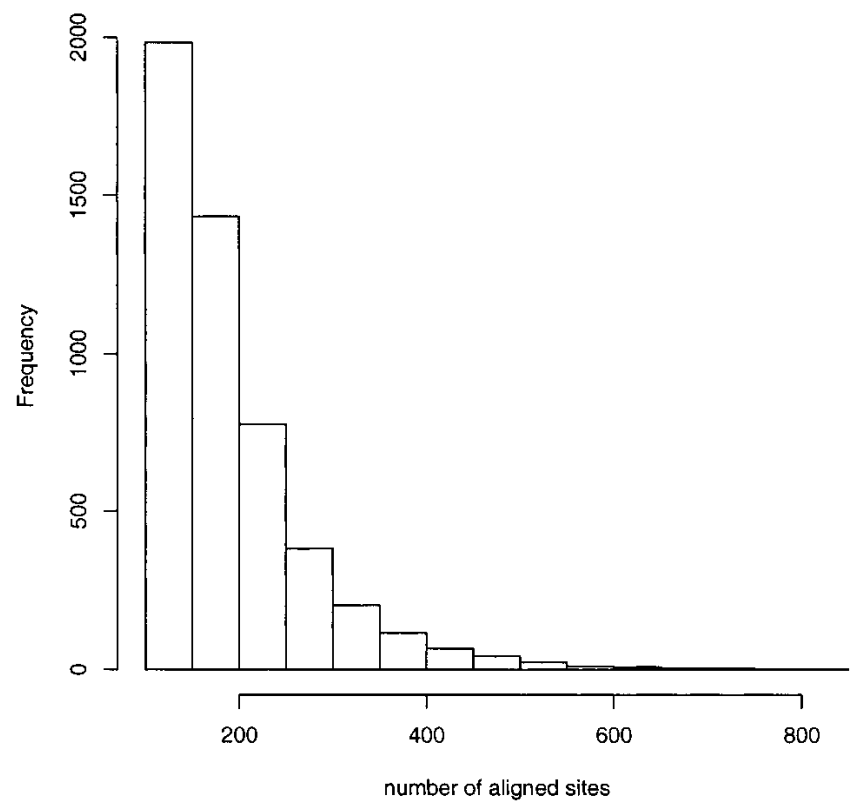

Figure 3 Histogram of the number of aligned sites in the evolutionary hotspots.

One of the clearcut results is that the rat branch length is slightly longer than the mouse branch. This finding is true not only on average, but also locally across the genomes. Furthermore, this observation is confirmed by independent analyses on different alignments (Cooper et al. 2004; Yang et al. 2004). The mutually exclusive ancient repeats and rodent-specific DNA yielded two interesting findings: First, their respective substitution rate matrices are very similar, indicating that perhaps the rodent substitution processes are similar in these two regions. This is consistent with the view that both types of regions are evolving neutrally. Second, the discrepancy between the mouserat distances inferred from the two regions, which is quite robust to the selection criteria, seems to be fairly strong evidence that the rodent-specific DNA evolved at a faster rate than the ancient repeats. Although this suggests that there is no single average neutral rate of substitution, it is important to bear in mind that there is still no clear computational assay for identifying neutrally evolving DNA. Because blocks were conservatively selected, both distance estimates are underestimates, and one may argue that the discrepancy is not inconsistent with the existence of a common, neutral, rate of substitution.

We showed that the relatively simple HKY model worked almost as well as the general REV model, and much better than the simplest JC model. This confirms the well-known observation that base composition and substitution bias should be taken into account in branch length estimation. Furthermore, given that the HKY model is simpler than the REV model, our finding suggests that using HKY for branch length estimation can be a workable compromise between accuracy and speed when analyzing large data sets.

The close distance of rat to its common ancestor with mouse means that the total predictive power of the mouse and rat genomes for identifying conserved regions in the human genome is not that much greater than using the mouse alone. Nevertheless, as we have pointed out, treating human as an outgroup to two similar genomes (mouse and rat), allows for the targeted identification of regions in the rodent genomes that are evolving in unexpected ways. We believe that the 5055 blocks we have identified represent a conservative estimate of the number of such regions. Because we only selected hotspots with at least 100 aligned sites, we necessarily miss the shorter ones. Perhaps one way to identify them is by sliding a window along a conserved region to detect very different rodent branch lengths.

It is important to note that the cutoffs used in our methodology are motivated partly by analyses of the average substitution patterns in the human, mouse, and rat lineages. Although the estimates depend on the thresholds used, and also on the alignment, systemic patterns do emerge from independent analyses, and the universal observation that the rat lineage is evolving faster than the mouse is confirmation of that. Thus, we believe that our identified hotspots do represent biologically interesting regions, and are not merely artifacts of selected parameters and heuristic cutoffs.

The natural generalization of our study is to identify regions in a multiple alignment where the inferred tree differs substantially from the consensus tree for the genome. Recent work by Billera et al. (2001) describes the "space of trees" that has unique geodesics, and thus provides a natural framework for quantitatively assigning a distance to a pair of trees. Our method on analyzing the rodent genomes for hotspots should extend to multiple organisms using this approach.

\section{METHODS}

\section{Alignment}

A multiple alignment of the human, mouse, and rat genomes was generated by first constructing a three-way homology map between the genomes and then aligning the homologous regions with MAVID (Bray and Pachter 2003; http://babson.math. berkeley.edu/mavid). Further details are in the companion paper describing MAVID (Bray and Pachter 2004).

\section{Ancient Repeats}

The locations of the human repetitive elements were downloaded from the UCSC Genome Browser (http://genome.ucsc. edu); simple and low-complexity repetitive elements were removed.

\section{Exons}

The locations of the human RefSeq exons were downloaded from the UCSC Genome Browser.

\section{Rodent-Specific DNA and Conserved Regions}

The criteria for rodent-specific DNA and conserved regions are more complicated because unlike the ancient repeats and exons, they have no well-defined positions. Intuitively, a block should have many aligned sites and few gaps. Our filters reflect this idea, and are similar to that used in (Yap and Speed 2003).

\section{Evolutionary Hotspots}

The 0.25 substitutions per site cutoff for the human branch length is natural, considering that this is a good threshold for separating exon from AR (Table 1). A more in-depth analysis of long exons and ancient repeats confirms this choice (Fig. 4). The

Table 3. Observed (Expected) Fractions of Hotspots in RefSeq Genes, RefSeq Exons, and ESTs

\begin{tabular}{lcc}
\hline Species & Mouse & Rat \\
\hline RefSeq genes & $39 \%(18 \%)$ & $17 \%(8 \%)$ \\
RefSeq exons & $2 \%(1 \%)$ & $2 \%(0.3 \%)$ \\
ESTs & $73 \%(37 \%)$ & $49 \%(18 \%)$ \\
\hline
\end{tabular}

The smaller expected fractions for rat are due to the fact that the rat databases are less complete. 

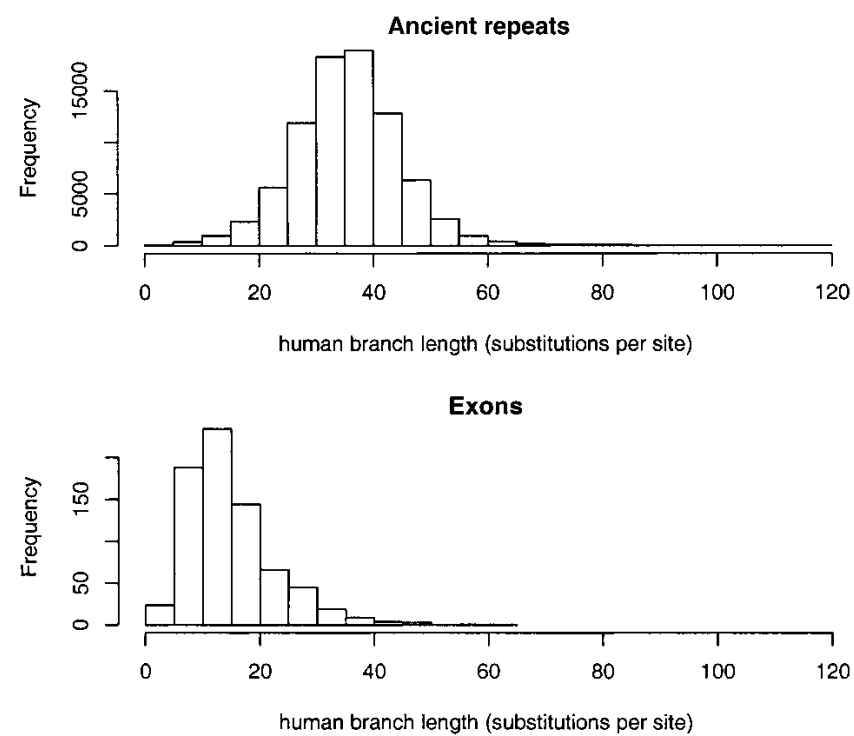

Figure 4 Histograms of the human branch length of blocks with at least 100 aligned sites from ancient repeats and exons. Branch lengths were estimated from each block individually without aggregation. A rough cutoff for separating the two distributions is 0.25 substitutions per site.

choice to impose the cutoff on the human branch is not arbitrary. Because human is much more distant from both rodents than they are from each other, a block with a short human branch is a strong signal of conservation. On the other hand, selecting blocks with rodent branches shorter than 0.052 (mouse) and 0.055 (rat) substitutions per site gives only 3820 hotspots, 1501 of which have human branch longer than 0.25 substitutions per site.

\section{Estimation}

To obtain the average substitution rates and branch lengths in Tables 1 and 2, we aggregated the blocks over the whole-genome alignment. We also applied the estimation procedure on individual blocks for some blocks in the ancient repeats and exons (Fig. 4) and for all conserved regions. Because aggregation is equivalent to gluing alignments into a long alignment, it suffices to explain the estimation procedure on a single block.

Let $\mathbf{h}, \mathbf{m}$, and $\mathbf{r}$ be the respective sequences from human, mouse, and rat. Assuming, as usual, that there was a common ancestor to human, mouse, and rat, which then split into a human lineage and a rodent lineage, which, in turn, split into mouse and rat, we then have a rooted phylogenetic tree relating the sequences as depicted in Figure 1 . The branch lengths $t_{1}, t_{2}$, $t_{3}$, and $t_{4}$ represent the evolutionary distances between the appropriate sequences, measured in the number of evolutionary events that occurred, averaged over all possible substitution paths. Because the rate of evolution is likely to have varied across lineages, there is no general correspondence between the evolutionary distances and the chronological time intervals.

The REV rate matrix $Q$ can be represented as

$$
\begin{array}{ccccc} 
& A & C & G & T \\
A & \cdot & \alpha \pi_{C} & \beta \pi_{G} & \gamma \pi_{T} \\
C & \alpha \pi_{A} & \cdot & \delta \pi_{G} & \epsilon \pi_{T} \\
G & \beta \pi_{A} & \delta \pi_{C} & \cdot & \zeta \pi_{T} \\
\mathrm{~T} & \gamma \pi_{A} & \epsilon \pi_{C} & \zeta \pi_{G} & \cdot
\end{array}
$$

The off-diagonal entries, the instantaneous substitution rates, are all nonnegative, and the diagonal entries are such that each row sums to $0 . \pi=\left(\pi_{A}, \pi_{C}, \pi_{G}, \pi_{T}\right)$ is the equilibrium distribution. Equivalently, $Q=R \Pi$, where $\Pi$ is diagonal and $R$ is symmetric:

$$
R=\left(\begin{array}{cccc}
\pi_{A} & 0 & 0 & 0 \\
0 & \pi_{C} & 0 & 0 \\
0 & 0 & \pi_{G} & 0 \\
0 & 0 & 0 & \pi_{T}
\end{array}\right),
$$

If $\alpha=\gamma=\delta=\zeta$ and $\beta=\epsilon$, then $Q$ is an HKY matrix. If $\pi$ is uniform and $\alpha=\beta=\gamma=\delta=\epsilon=\zeta$, then $Q$ is a JC matrix. Finally, if $\pi_{A}=\pi_{T}$, $\pi_{C}=\pi_{G}, \alpha=\zeta$ and $\beta=\epsilon$, then the substitution rates are invariant under complementation; for example, $Q(A, C)=Q(T, G)$. In this case, $Q$ is called strand-symmetric.

Suppose that $Q$ is calibrated, that is,

$$
\sum_{i} \hat{\pi}(i) Q(i, i)=-1
$$

or equivalently, the average number of substitutions per unit of evolutionary time is 1 . The transition matrix $P_{t}$ is given by

$$
P_{t}=\exp (Q t)
$$

The probability that an aligned site has human, mouse, and rat bases $a, b$, and $c$ (see Fig. 1 ) is obtained by summing over all possible ancestral bases:

$$
\begin{aligned}
\operatorname{Pr}(a, b, c) & =\sum_{z} \sum_{y} \pi(z) P_{t_{1}}(z, a) P_{t_{2}}(z, y) P_{t_{3}}(y, b) P_{t_{4}}(y, c) \\
& =\sum_{z} \sum_{y} \pi(y) P_{t_{2}}(y, z) P_{t_{1}}(z, a) P_{t_{3}}(y, b) P_{t_{4}}(y, c) \\
& =\sum_{y} \pi(y) P_{t_{1}+t_{2}}(y, a) P_{t_{3}}(y, b) P_{t_{4}}(y, c) .
\end{aligned}
$$

Two consequences of reversibility are reflected in this expression. First, the joint probability is the same as if the rodent ancestor were the root, simplifying the calculation. Second, $t_{1}$ and $t_{2}$ are not estimable from the multiple alignment, although their sum is. We shall refer to the human, mouse, and rat branch lengths as

$$
\begin{aligned}
t_{h} & =t_{1}+t_{2} \\
t_{m} & =t_{3}, \\
t_{r} & =t_{4} .
\end{aligned}
$$

Thus, we are forced to estimate branch lengths of an unrooted tree with three leaves, which is a star tree. Note that the human branch is just a mathematical convenience, but the rodent branches correspond to true lineages.

Assuming site independence and homogeneity, the probability of a subalignment (without gaps) is the product of the site-specific probabilities. This is maximized numerically to obtain estimates of the rate matrix and the branch lengths. By suitably constraining the rate matrix, we get the maximum likelihood (ML) estimates of an REV, HKY, or JC rate matrix. The whole-genome analysis for REV could be finished within $7 \mathrm{~h}$ on a single 2.6-Ghz processor.

\section{ACKNOWLEDGMENTS}

We thank Nicolas Bray and Colin Dewey for generating the whole-genome alignments; and Greg Cooper, Ross Hardison, David Haussler, Webb Miller, and Arend Sidow for extensive discussions. Special appreciation goes to Krishna Roskin for reconciling the findings of the different groups. We also thank the referees for many helpful comments and suggestions. L.P. and V.B.Y. were partially funded by a grant from the NIH (R01HG02362-01).

The publication costs of this article were defrayed in part by payment of page charges. This article must therefore be hereby 
marked "advertisement" in accordance with 18 USC section 1734 solely to indicate this fact.

\section{REFERENCES}

Billera, L.J., Holmes, S.P., and Vogtmann, K. 2001. Geometry of the space of phylogenetic trees. Adv. Appl. Math. 27: 733-767.

Boffelli, D., McAuliffe, J., Ovcharenko, D., Lewis, K.D., Ovcharenko, I., Pachter, L., and Rubin, E.M. 2003. Phylogenetic shadowing of primate sequences to find functional regions of the human genome. Science 299: 1391-1394.

Bray, N. and Pachter, L. 2003. The MAVID multiple alignment server. Nucleic Acids Res. 31: 3525-3526.

. 2004. MAVID: Constrained ancestral alignment of multiple sequences. Genome Res. (this issue).

Cooper, G.M., Brudno, M., NISC Comparative Sequencing Program, Green, E.D., Batzoglou, S., and Sidow, A. 2003. Quantitative estimates of sequence divergence. Genome Res. 13: 813-820.

Cooper, G.M., Brudno, M., Stone, E.A., Dubchak, I., Batzoglou, S., and Sidow, A. 2004. Characterization of evolutionary rates and constraints in three mammalian genomes. Genome Res. (in press).

Dubchak, I., Brudno, M., Loots, G.G., Pachter, L., Mayor, C., Rubin, E.M., and Frazer, K.A. 2000. Active conservation of noncoding sequences revealed by three-way species comparison. Genome Res. 10: $1304-1306$.

Hardison, R.C., Roskin, K.M., Yang, S., Dickhans, M., Kent, W.J., Weber, R., Elnitski, L., Li, J., O'Connor, M., Kolbe, D., et al. 2003. Covariation in frequencies of substitution, deletion, transposition, and recombination during eutherian evolution. Genome Res. 13: $13-26$.

Lee, I.Y., Westway, D., Smit, A.F.A., Wang, K., Seto, J., Chen, L., Acharya, C., Ankener, M., Baskin, D., Cooper, C., et al. 1998. Complete genome sequence and analysis of prion protein gene region from three mammalian species. Genome Res. 8: 1022-1037.

Miller, W. 2001. Comparison of genomic DNA sequences. Bioinformatics 17: $391-397$

Moret, B.M.E., Wang, L.-S., Warnow, T., and Wyman, S. 2001. New approaches for reconstructing phylogenies based on gene order Bioinformatics 17 Suppl. 1: S165-173.

Rat Genome Sequencing Project Consortium. 2004. Genome sequence of the Brown Norway Rat yields insights into mammalian evolution. Nature (in press).

Tavaré, S. 1986. Some probabilistic and statistical problems in the analysis of DNA sequences. Lectures on Mathematics in the Life Sciences 17: $57-86$.

Thomas, J.W., Touchman, J.W., Blakesley, R.W., Bouffard, G.G., Beckstrom-Sternberg, S.M., Margulies, E.H., Blanchette, M., Siepel, A.C., Thomas, P.J., McDowell, J.C., et al. 2003. Comparative analysis of multi-species sequences from targeted genomic regions. Nature 424: 788-793.

Waterston, R.H., Lindblad-Toh, K., Birney, E., Rogers, J., Abril, J.F., Agarwal, P., Agarwala, R., Ainscough, R., Alexandersson, M., An, P., et al. 2002. Initial sequencing and comparative analysis of the mouse genome. Nature 420: $520-562$.

Wiehe, T., Guigo, R., and Miller, W., 2000. Genome sequence comparisons: Hurdles in the fast lane to functional genomics. Briefings in Bioinformatics 4: 381-388.

Yang, S., Schwartz, S., Chiaromonte, F., Roskin, K.M., Haussler, D., Miller, W., and Hardison, R.C. 2004. Patterns of insertions and their covariation with substitutions in the rat, mouse and human genomes. Genome Res. (this issue).

Yang, Z. 1994. Estimating the pattern of nucleotide substitution. J. Mol. Evol. 39: 105-111.

Yap, V.B. and Speed, T.P. 2004. Modeling DNA base substitution in large genomic regions from two organisms. J. Mol. Evol. 58: 12-16.

\section{WEB SITE REFERENCES}

http://baboon.math.berkeley.edu/hotrodent/; rodent hotspots. http://baboon.math.berkeley.edu/mavid/; MAVID alignment server. http://genome.ucsc.edu/; UCSC Genome Bioinformatics.

Received September 11, 2003; accepted in revised form December 27, 2003. 